\title{
Esfuerzo, captura y captura por unidad de esfuerzo en la pesquería de la langosta de Juan Fernández (Jasus frontalis), durante la temporada de pesca 1996-1997*
}

\author{
Patricio M. Arana y Rodrigo Vega \\ Escuela de Ciencias del Mar, Universidad Católica de Valparaíso \\ Casilla 1020, Valparaíso, Chile \\ E-mail: parana@ucv.cl
}

Recibido: 7 diciembre 1998; versión corregida: 30 junio 1999; aceptado: 10 septiembre 1999

\begin{abstract}
RESUMEN Se analiza el esfuerzo, las capturas y los rendimientos en la pesquería de la langosta de Juan Fernández (Jasus frontalis), durante la temporada de pesca 1996-1997. Con este fin se emplea información mensual proporcionada por los pescadores en formularios implementados con tal objetivo en las islas Robinson Crusoe-Santa Clara, Alejandro Selkirk e islas Desventuradas (islas San Félix y San Ambrosio). Esta información se complementó con registros obtenidos directamente por personal técnico a bordo de las embarcaciones artesanales que operan en torno a las islas Robinson Crusoe y Santa Clara. Estos últimos permitieron reunir antecedentes referidos a captura por trampa por zona de pesca y profundidad.

En la temporada analizada el desembarque total de este recurso alcanzó a 53.543 unidades (50 ton). De ese total, 26.219 ejemplares (49\%) se extrajeron en torno a las islas Robinson Crusoe y Santa Clara, 17.929 (33\%) en la isla Alejandro Selkirk y $9.395(18 \%)$ en las islas Desventuradas. En el primer grupo de islas mencionado, el porcentaje de individuos comerciales sobre el total capturado alcanzó al 7,5\%. Los rendimientos de ejemplares comerciales variaron mensualmente entre 0,53 y 0,59 langostas/trampa, con los valores más altos de 112 a $137 \mathrm{~m}$ de profundidad (61 y 75 bz) y en las zonas ubicadas al sur y sur-oeste de las islas. El esfuerzo, definido como el número total de trampas caladas, se concentró en el área sur-oeste de la isla Santa Clara. Las trampas son caladas al comienzo de la temporada preferentemente cerca de la costa y a partir de diciembre son desplazadas hacia aguas de mayor profundidad, concentrándose los aparejos al final de la temporada entre 112 y 165 m $(61$ y 90 bz).

Comparativamente los rendimientos más altos de ejemplares comerciales fueron obtenidos en las islas Desventuradas, donde se capturó como promedio 174 langostas/salida; rendimiento notablemente superior al registrado en la isla Alejandro Selkirk, de 31,6 langostas/salida y en las islas Robinson Crusoe-Santa Clara, de 10,2 langostas/salida.
\end{abstract}

Palabras claves: esfuerzo, captura, CPUE, langosta, Jasus frontalis, archipiélago de Juan Fernández, Chile.

\section{Effort, catch and catch per unit of effort in the Juan Fernandez spiny rock lobster fishery (Jasus frontalis), during the 1996-1997 fishing season*}

\begin{abstract}
ABSIRACT. The effort, catches and yields of the Juan Fernandez spiny rock lobster (Jasus frontalis), during the 1996-1997 fishing season. For this purpose the monthly information given by the fishermen in ad-hoc forms was used in the islands Robinson Crusoe-Santa Clara, Alejandro Selkirk and Desventuradas (San Félix and San Ambrosio islands). This information was complementary records obtained directly by technical personnel on board of artisanal boats operating off Robinson Crusoe and Santa Clara islands. The latter enabled us to gather antecedents referred to the catch per trap by fishing zone and depth.
\end{abstract}

* Artículo generado como parte del proyecto "Análisis y evaluación indirecta de la pesquería de langosta de Juan Fernández" (Proyecto FIP 96/22), financiado por el Fondo de Investigación Pesquera y realizado por la Universidad Católica de Valparaíso. 
For the analyzed season, the total landings of this resource reached 53,543 units (50 ton). From the total, 26,219 specimens (49\%) were extracted around Robinson Crusoe and Santa Clara islands, 17,929 (33\%) in Alejandro Selkirk island and 9,395 (18\%) from Desventuradas islands. For the first group of islands, the percent of commercial lobster over the total catch reached $7.5 \%$. The yields of commercial specimens varied monthly between 0.53 y 0.59 lobsters/ trap, with the maximum values between 112 and $137 \mathrm{~m}$ depth (61 y 75 fathoms) and in zones located to the south and southwest of the islands. The effort, as the number of total traps settled, was concentrated in the southwest area of Santa Clara island. Traps are settled at the beginning of the season preferentially near the coast and, early in December, they are displaced towards greater depth waters, concentrating the traps towards the end of the season between 112 and $165 \mathrm{~m}$ (61 y 90 fathoms).

Comparatively, the maximun average yield of 174 lobsters per trip off Desventuradas islands were remarkable higher than the average yield, of 31.6 and 10.2 lobsters per trip from Alejandro Selkirk island and Robinson Crusoe-Santa Clara islands, respectively.

Key words: effort, catch, CPUE, spiny rock lobster, Jasus frontalis, Juan Fernández archipelago, Chile.

\section{INTRODUCCIÓN}

Entre los recursos pesqueros que destacan por su aceptación y valor comercial se encuentran las langostas marinas, crustáceos que en Chile están representados por las familias Scyllaridae y Palinuridae. Entre estas últimas están descritas las especies Panulirus pascuensis Reed, 1954 presente en las islas de Pascua y Salas y Gómez, Jasus frontalis H. Milne-Edwards, 1837 en el archipiélago de Juan Fernández e islas Desventuradas (islas San Félix y San Ambrosio) y Projasus bahamondei George, 1976 en montañas submarinas del Pacífico suroriental, esta última capturada en forma ocasional cerca de la costa de América del Sur.

De ellas, la langosta de Juan Fernández es la especie de mayor importancia socioeconómica, ya que su extracción representa la principal actividad realizada por los habitantes de dicho grupo insular, explotación que se ha realizado en forma continua desde 1893 (Arana, 1987). Este recurso se distribuye batimétricamente entre los 2 y 180 metros de profundidad y se le captura utilizando trampas rectangulares construidas artesanalmente con maderas locales, las que están compuestas de dos cámaras, cada una con una entrada cónica hacia el interior denominada "buche". La actividad extractiva de este crustáceo es realizada por pescadores artesanales, que utilizan embarcaciones semejantes a botes balleneros de doble proa (8-10 m de eslora), equipadas con motores fuera de borda alojados en un sistema de cajón interno, clasificadas genéricamente como "chalupas de alta mar".

El desembarque de los últimos 65 años, presenta una pronunciada variación anual, producto de diversos factores tales como: cambios naturales en la abundancia del recurso, factores ambientales que pueden favorecer o perjudicar determinadas clases anuales, condiciones meteorológicas que influencian el esfuerzo pesquero y la realización en forma esporádica de operaciones extractivas en las islas Desventuradas (Arana, 1987). Si bien entre 1952 y 1964 se registraron desembarques de alrededor de 120 ton/ año, en los años posteriores se observó una constante disminución, alcanzando en la temporada de pesca 1979-1980 sólo un décimo del nivel antes mencionado. A partir de 1981 y hasta 1987 las capturas anuales presentan un leve aumento y luego se observa que nuevamente se reducen en forma progresiva, llegando a 36 ton en 1996 (SERNAPESCA, 1998). Una posible explicación para la deprimida situación en que se encuentra esta pesquería sería el incremento excesivo del esfuerzo pesquero, representado tanto en el número de trampas utilizadas como por la eficiencia individual de cada una de ellas (Arana, 1983). Así, también, puede haber influido en esta situación la extracción de ejemplares bajo la talla mínima legal de desembarque, aspecto que no está debidamente cuantificado en cantidad ni el efecto negativo que puede tener en la conservación de este recurso (Silva y Cerda, 1984, Arana, 1985, 1987).

En este ámbito, en los últimos 25 años el estado de esta pesquería se ha investigado solamente en algunas temporadas. Por ello, su evolución se ha descrito esporádicamente, mediante trabajos que han registrado el esfuerzo ejercido, estableciendo la distribución espacial de las trampas y los rendimientos que se obtienen de acuerdo a la zona de pesca y al rango de profundidad en que se efectúan las capturas (Arana y Melo, 1973; Arana y Toro, 1985 y Henríquez et al., 1985). Sin embargo, se hace necesario mantener continuidad en este tipo de investigaciones, que no sólo determinen fluctuaciones den- 
tro del período de estudio, sino que además permitan visualizar la evolución de la pesquería en el tiempo y de esta forma evaluar la eficiencia de las medidas de manejo vigentes.

El presente trabajo tiene como principal objetivo determinar la captura y el esfuerzo de pesca ejercido en la pesquería de langosta en el archipiélago de Juan Fernández e islas Desventuradas durante la temporada de pesca 1996-1997. Así, también, tiene por finalidad calcular la captura por unidad de esfuerzo (CPUE) por zona de pesca y estrato de profundidad en torno a las islas Robinson Crusoe y Santa Clara con el propósito de describir la evolución que han tenido estas variables en el tiempo.

\section{MATERIALES Y MÉTODOS}

\section{Aspectos generales}

Con el fin de conocer el esfuerzo, las capturas y los rendimientos que se obtienen en la pesquería de la langosta de Juan Fernández, se procedió a reunir mensualmente información de las actividades extractivas realizadas durante la temporada de pesca 1996-1997. En general se siguió los mismos procedimientos de recopilación de información utilizados anteriormente en torno a las islas Robinson Crusoe y Santa Clara, durante la temporada 19711972 por Arana y Melo (1973), en 1980-1981 por Arana y Toro (1985), así como por el Instituto de Fomento Pesquero en 1984-1985 (Henríquez et al., 1985). Todo ello con el objeto de hacer comparable los resultados y poder establecer la evolución de esta pesquería en el tiempo.

El registro de las capturas se efectuó considerando el número de ejemplares extraídos, tomando para ello en cuenta el sistema de trabajo utilizado en forma tradicional en esta pesquería. El esfuerzo fue considerado de acuerdo a la cantidad de embarcaciones en operación, así como del número de salidas realizadas y trampas utilizadas.

Por otra parte, es preciso señalar que en esta pesquería, por el hecho de estar vigente una talla mínima legal de comercialización de $115 \mathrm{~mm}$ de longitud cefalotorácica, medida desde la base de las antenas (equivalente a $98 \mathrm{~mm}$ si se miden los ejemplares desde el arco postocular) y la prohibición de desembarcar hembras ovíferas, los ejemplares son seleccionados inmediatamente después de ser extraídos y sólo aquellos que cumplen con la normativa pesquera son retenidos por los pescadores $(=$ ani- males comerciales). Los restantes son devueltos al mar en el mismo lugar en que fueron capturados (Arana, 1985). Por tal razón, los análisis se efectuaron considerando dos fuentes de información: los desembarques y las capturas en el mar. En este último caso, se empleó personal que realizó los muestreos a bordo de las embarcaciones que operan en la pesquería con el objeto de obtener la información requerida para los análisis, antes que sean seleccionadas las langostas que serán desembarcadas, del total de ejemplares capturados en cada trampa que se revisa. Esto permitió también establecer la fracción comercial, respecto del total de ejemplares capturados y determinar los rendimientos por zona y por estrato de profundidad.

De acuerdo a los planteamientos antes señalados, la determinación del esfuerzo, captura y captura por unidad de esfuerzo (CPUE) se realizó de acuerdo a dos aproximaciones metodológicas. La primera de ellas consideró la información global proporcionada por los pescadores, aunque sin referencia a zona y profundidad, la cual fue denominada para fines de análisis como Desembarques. La segunda, fue obtenida directamente por personal técnico embarcado en las chalupas de alta mar que explotan este recurso, los que obtienen registros de cada una de las trampas revisadas, la que fue identificada como Capturas en el mar.

\section{Desembarques}

Esta información es proporcionada por los pescadores mediante el formulario implementado por el Servicio Nacional de Pesca (SERNAPESCA), que es entregado mensualmente a la Capitanía de Puerto de Juan Fernández, entidad que lo recepciona y posteriormente lo envía a la Dirección Regional de SERNAPESCA V Región. En esos formularios los patrones de cada embarcación indican la fecha en que realizaron las salidas de pesca y consignan las unidades de langosta capturadas en cada una de ellas.

Mediante estos formularios también es posible establecer el número de botes en operación, el número de salidas de pesca y las langostas desembarcadas por cada bote. Paralelamente, en cada mes se encuestó a todos los patrones de las embarcaciones que operan en las islas Robinson Crusoe y Santa Clara, a fin de conocer el número de trampas que estaba calando, en atención a que dicha variable no queda consignada en los formularios antes descritos. Con todos estos registros, es posible determinar la captura total en el respectivo mes y la CPUE por bote, por salida y por trampa. En este último caso, 
se considera el número teórico de aparejos revisados mensualmente por cada bote, que es calculado como la cantidad de trampas que utiliza cada embarcación, multiplicado por el número de salidas efectuado en el respectivo mes.

\section{Capturas en el mar}

Como se señaló precedentemente, la forma más adecuada para obtener y analizar los rendimientos de pesca, corresponde a la captura en número de individuos, obtenida por trampa efectivamente revisada. Estas labores fueron realizadas por el personal técnico embarcado, únicamente en torno a las islas Robinson Crusoe y Santa Clara.

En la correspondiente salida se registró la ubicación (zona de calado) de cada trampa mediante demarcaciones a tierra y la profundidad por la longitud del orinque de cala, como así también la captura de ejemplares comerciales y no comerciales, de acuerdo a la selección efectuada por el patrón de la embarcación. Complementariamente, se midió la longitud cefalotorácica (distancia en línea recta medida desde el arco postocular hasta el límite posterior del caparazón) a todos los ejemplares capturados con el objeto de establecer la estructura del stock. Estas mediciones se realizaron utilizando medidores vernier con precisión de $0,1 \mathrm{~mm}$.

El proceder de esta manera, posibilitó distinguir tres valores de CPUE: uno de ellos referido al número total de ejemplares retenidos en cada trampa virada; otro, que considera solamente los organismos que es permitido comercializar y un tercer indicador, que incluye únicamente la extracción de individuos que deben ser devueltos al mar (ejemplares bajo la talla mínima de extracción y hembras ovíferas).

Para establecer posibles diferencias en los rendimientos de acuerdo a la profundidad de calado de las trampas, la información fue agrupada en nueve estratos de profundidad, de 15 brazas cada uno, en atención a que ésta es la unidad de medida de uso común entre los pescadores de la zona. Dichos rangos de profundidad equi- valen a: 0-27, 29-55, 57-82, 84-110, 112-137, 139-165, 167$192,194-220$ y $222-247 \mathrm{~m}$.

Asimismo, con el objeto de establecer posibles diferencias en los rendimientos en torno a estas islas, el área de estudio se dividió en 10 zonas de trabajo. Estas zonas son coincidentes con las utilizadas en anteriores investigaciones realizadas sobre esta misma especie (Arana y Pizarro, 1971; Arana y Melo, 1973; Arana y Toro, 1985; Larraín y Yañez, 1985; Henríquez et al., 1985). Estas zonas quedan definidas en torno a ambas islas, en sentido horario, comenzando desde Bahía Cumberland (zona 1), donde se ubica el pueblo de San Juan Bautista, puerto base de las embarcaciones que operan en las islas Robinson Crusoe y Santa Clara (Fig. 1). La denominación de estas zonas se indica a continuación:

\begin{tabular}{|c|l|l|}
\hline Zona & \multicolumn{1}{|c|}{ Nombre } & \multicolumn{1}{|c|}{ Límites locales } \\
\hline 1 & Bahía Cumberland & El Portal - Punta Bacalao \\
2 & Puerto Francés & Punta Bacalao - Los Toros \\
3 & Playa Larga & Los Toros - Las Rosas \\
4 & Bahía Villagra & Las Rosas - Islote Vinillo \\
5 & La Canal & Islote Vinillo - Las 400 \\
& & (Entre islas Robinson Crusoe \\
& & y Santa Clara) \\
6 & Santa Clara & Al sur y suroeste de la \\
7 & Bsla Santa Clara \\
8 & Bahía del Padre & Las 400 - El Pito \\
9 & Islote Juanango & El Pito - Cueva de los Jureles \\
10 & La Vaquería & El Ancla - El Portal \\
\hline
\end{tabular}

\section{Análisis de la información}

Los registros de Desembarques obtenidos en las islas Robinson Crusoe-Santa Clara, Alejandro Selkirk e islas Desventuradas se consideraron separadamente con el fin de determinar en cada uno de estos lugares la captura total mensual, el número total de botes en operación y el número de salidas realizadas. Con excepción de la isla Alejandro Selkirk, en los otros dos grupos de islas se determinó mediante encuestas, el número de trampas utilizadas individualmente en cada chalupa de alta mar y en toda la flota. Con dichos valores fue posible determinar en esos lugares los rendimientos promedio por bote, por salida y por trampa, de acuerdo al número teórico de aparejos revisados cada mes.

En el caso particular de las Capturas en el mar, la información fue agrupada por mes, por zona y por estrato de profundidad con el objeto de determinar la distribución espacial del esfuerzo y las capturas, y precisar su dinámica temporal. 


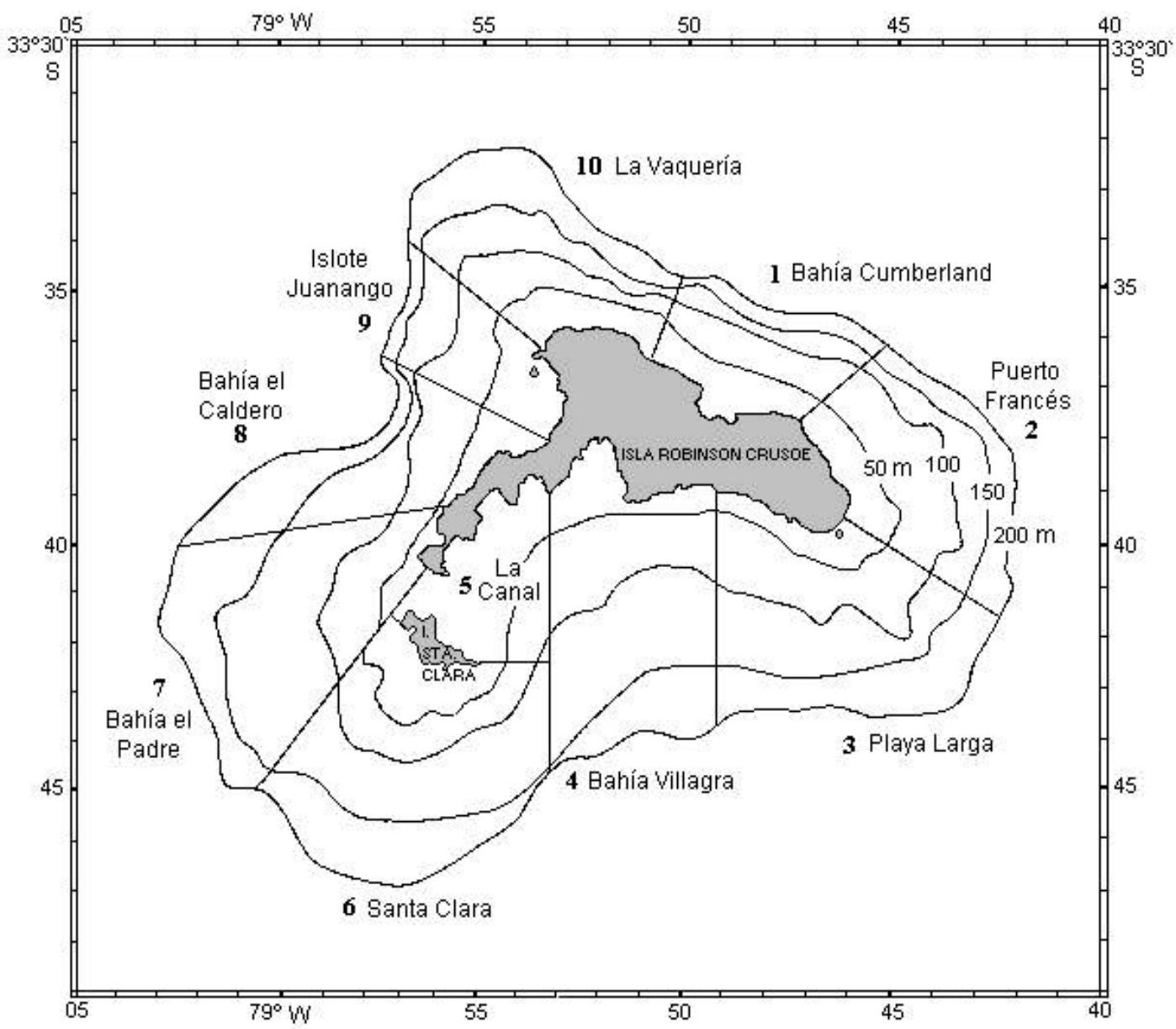

Figura 1. Area de estudio y zonas de pesca utilizadas para la recolección de información biológico-pesquera.

Figure 1. Area of study and fishing zones used for gathering of fisheries-biological information.

En este caso, para el cálculo de los rendimientos se utilizó como unidad de esfuerzo la operación individual de cada una de las trampas, correspondiendo la CPUE al número de langostas retenidas por trampa revisada (langostas/trampa).

Con la finalidad de determinar posibles diferencias en los rendimientos de langostas comerciales, tanto por zona de pesca como por estrato de profundidad y entre los meses, la información se analizó mediante el Modelo estadístico factorial con una observación por celda (Montgomery, 1991). El modelo utilizado fue el siguiente:

$$
Y_{i j}=\mu+t_{1}+b_{j}+e_{i j} \quad \begin{aligned}
& i=1,2, \ldots, a \\
& j=1,2, \ldots, b
\end{aligned}
$$

donde:

$\mathrm{Y}_{\mathrm{ij}} \quad$ : observación de la respuesta para el tratamiento $(\mathrm{i}, \mathrm{j})$

$\mu \quad:$ media global

$t_{i} \quad$ : efecto sobre la respuesta debido al i-ésimo nivel del factor A (mes)

$\mathrm{b}_{\mathbf{j}} \quad$ : efecto sobre la respuesta debido al j-ésimo nivel del factor B (zona de pesca o estrato de profundidad)

$e_{i j} \quad$ : error aleatorio del tratamiento $i, j$

Como prueba de significancia se desarrolló el Análisis de Varianza (ANDEVA), docimándose en cada oportunidad la no existencia de efectos individuales de los factores, es decir: 


$$
\begin{array}{lll}
\mathrm{H}_{0:} & \mathrm{t}_{\mathrm{i}}=\mathbf{0} & \mathrm{y} \\
\mathrm{H}_{0:} & \mathrm{b}_{\mathbf{j}}=\mathbf{0} & \text { para toda combinación } \mathrm{i}, \mathrm{j}
\end{array}
$$

Debido a la variabilidad en número de los registros de CPUE, para el análisis estadístico de la información se utilizó solamente un valor por celda, correspondiente ésta a la media aritmética de las observaciones de cada categoría. Del mismo modo, en el análisis se utilizaron sólo aquellos bloques de información que no presentaron valores faltantes.

\section{RESULTADOS}

\section{Aspectos generales}

Las actividades de pesca de langosta en torno a las islas Robinson Crusoe y Santa Clara, efectuadas por un máximo de 40 embarcaciones y aproximadamente 90 pescadores, fueron realizadas en el período autorizado para la extracción de este recurso, es decir, entre el $1^{\circ}$ de octubre de 1996 y el 14 de mayo de 1997. En la isla Alejandro Selkirk, entre septiembre de 1996 y abril de 1997 realizó actividades extractivas un máximo de 11 botes (28 pescadores). Finalmente, en las islas Desventuradas se efectuaron operaciones pesqueras con tres embarcaciones y 8 pescadores que se desplazaron desde isla Alejandro Selkirk, entre el 16 de noviembre y el 14 de diciembre de 1996.
Entre septiembre de 1996 y mayo de 1997, la extracción total de langosta de Juan Fernández fue de 53.543 unidades, que representa una captura en peso de alrededor de 50 ton. De ese total, 26.219 ejemplares (49\%) se capturaron en torno a las islas Robinson Crusoe y Santa Clara, 17.929 langostas (33\%) en la isla Alejandro Selkirk y 9.395 individuos en las islas Desventuradas (18\%) (Tabla 1).

En las islas Robinson Crusoe y Santa Clara, el número de botes que operó mensualmente fluctuó entre 35 y 40, cantidades registradas en octubre de 1996 y febrero de 1997, respectivamente (Tabla 2). Esta variación es explicada en parte por el traslado de algunos botes entre esas islas y Alejandro Selkirk, así como también por la paralización de actividades de algunas embarcaciones antes de finalizar la temporada de pesca.

El número mensual de viajes de pesca estuvo comprendido entre 376 salidas/mes (enero) y 124 salidas/mes (mayo) (Tabla 2). Por lo general, el número de viajes mensuales está directamente relacionado con la cantidad de botes en operación y las condiciones meteorológicas imperantes.

Otro aspecto relevante en esta pesquería tiene relación con la variación del esfuerzo ejercido en estas islas durante el transcurso de la temporada, ya que paulatinamente se disminuyó el número de trampas que utilizó cada bote. Los antecedentes recopilados durante la temporada analizada demuestran

Tabla 1. Unidades de langosta desembarcadas mensualmente entre septiembre de 1996 y mayo de 1997.

Table 1. Monthly landings (number of lobsters) between September 1996 and May 1997.

\begin{tabular}{|lcccc|}
\hline Mes & $\begin{array}{c}\text { Islas Robinson Crusoe } \\
\text { y Santa Clara }\end{array}$ & $\begin{array}{c}\text { Isla Alejandro } \\
\text { Selkirk }\end{array}$ & $\begin{array}{c}\text { Islas San Félix y } \\
\text { San Ambrosio }\end{array}$ & Total \\
\hline Septiembre & - & 2.303 & - & 2.303 \\
Octubre & 4.037 & 1.366 & - & 5.403 \\
Noviembre & 3.555 & 3.146 & 5.967 & 12.668 \\
Diciembre & 3.996 & 3.221 & 3.428 & 10.645 \\
Enero & 4.860 & 3.372 & - & 8.232 \\
Febrero & 4.384 & 1.516 & - & 5.900 \\
Marzo & 3.174 & 1.596 & - & 4.770 \\
Abril & 1.541 & 1.409 & - & 672 \\
Mayo & $672 *$ & - & $\mathbf{9 . 3 9 5}$ \\
\hline Total & $\mathbf{2 6 . 2 1 9}$ & $\mathbf{1 7 . 9 2 9}$ & $\mathbf{5 3 . 5 4 3}$ \\
\hline
\end{tabular}

(*) Valor amplificado al total de las embarcaciones que operaron en mayo

Fuente: Formularios de desembarque artesanal (SERNAPESCA) 
Tabla 2. Resumen de botes en operación, salidas de pesca y trampas caladas por mes (Islas Robinson Crusoe y Santa Clara).

Table 2. Summary of operating boats, fishing trips and settled traps by month (Robinson Crusoe and Santa Clara islands).

\begin{tabular}{|lccc|}
\hline Mes & Botes en operación & Salidas de pesca (*) & Trampas caladas (*) \\
\hline Octubre (1996) & 35 & 322 & $\mathrm{~s} / \mathrm{i}$ \\
Noviembre & 37 & 333 & $\mathrm{~s} / \mathrm{i}$ \\
Diciembre & 38 & 357 & 1.066 \\
Enero (1997) & 38 & 376 & 1.024 \\
Febrero & 40 & 344 & 1.016 \\
Marzo & 37 & 330 & 829 \\
Abril & 35 & 228 & 551 \\
Mayo & 35 & 124 & 404 \\
\hline
\end{tabular}

(*) Cuando no se contó con información de un bote, se utilizó la media mensual para incluirlo s/i Sin información Fuente: Formularios de desembarque artesanal (SERNAPESCA) e información propia

que el número de aparejos en uso alcanzó en diciembre a 1066 trampas, las cuales se redujeron progresivamente a tan sólo 404 trampas en operación en mayo (Tabla 2). La disminución en la cantidad de trampas caladas está relacionado por una parte con la pérdida de aparejos, los que no son reemplazados, y al retiro de trampas por parte de algunos pescadores que dan por finalizadas sus actividades antes que termine oficialmente la temporada de pesca.

\section{Desembarques}

Los registros sobre las capturas obtenidas y el esfuerzo aplicado en torno a las islas Robinson Crusoe y Santa Clara (octubre 1996-mayo 1997) consideró la totalidad de la flota artesanal en operación, con la única excepción de mayo. Durante ese último mes sólo se logró oficializar la entrega de una parte de los formularios de capturas mensuales, motivo por el cual los registros disponibles se amplificaron en forma proporcional, a fin de que éstos representaran a toda la flota extractiva.

En relación con el desembarque mensual de langostas en las islas Robinson Crusoe y Santa Clara, se observó que los mayores valores fueron registrados en octubre, enero y febrero, mientras que los menores se establecieron hacia el final de la temporada (abril-mayo) (Tabla 1). La captura por bote presenta valores promedios mensuales comprendidos entre 19,2 langostas/bote (mayo) y 127,9 langostas/ bote (enero), mientras que la captura promedio por viaje de pesca fluctuó entre 5,4 langostas/salida en mayo y 12,9 langostas/salida en enero (Tabla 3). La CPUE media por trampa teórica calada, entre diciembre de 1996 y mayo de 1997, presenta estimados relativamente constantes, con valores comprendidos entre 0,41 langostas/trampa en diciembre y 0,51 langostas/trampa en enero y mayo (Tabla 3 ).

Con los registros recopilados en la isla Alejandro Selkirk e islas Desventuradas, vale decir: desembarques, botes en operación y número de salidas, se logró establecer la notable diferencia en los rendimientos que se obtienen entre las islas. Estos fueron más altos en las islas Desventuradas, donde como promedio se capturaron 174 langostas/salida y en la isla Alejandro Selkirk, promedios mensuales entre 23,5 y 41,3 langostas/salida (Tablas 4 a 6).

\section{Capturas en el mar}

El programa de muestreo en el mar, realizado únicamente alrededor de las islas Robinson Crusoe y Santa Clara entre enero y mayo de 1997, reunió información proveniente de 215 salidas de pesca, determinándose de esta manera la ubicación mensual por zona de pesca y estrato de profundidad a un total de 3.126 trampas (Tablas 7 y 8). Además, se calculó el rendimiento de ejemplares totales, comerciales y no comerciales, referenciado mensualmente y por zona de pesca y profundidad de calado, considerando para ello un total de 22.919 ejemplares.

El porcentaje promedio de ejemplares comerciales varió mensualmente, con valores de $8,4 \%$ en 
Tabla 3. Captura por unidad de esfuerzo (CPUE) mensual por bote en operación, salida de pesca y trampa calada (Islas Robinson Crusoe y Santa Clara).

Table 3. Monthly catch per unit effort (CPUE) per operating boat, fishing trip and settled traps (Robinson Crusoe and Santa Clara islands).

\begin{tabular}{|lccc|}
\hline Mes & Langostas/bote & $\begin{array}{c}\text { CPUE } \\
\text { Langostas/salida }\end{array}$ & Langostas/trampa calada (*) \\
\hline Octubre (1996) & 115,3 & 12,5 & $\mathrm{~s} / \mathrm{i}$ \\
Noviembre & 96,1 & 10,7 & $\mathrm{~s} / \mathrm{i}$ \\
Diciembre & 105,2 & 11,2 & 0,41 \\
Enero (1997) & 127,9 & 12,9 & 0,51 \\
Febrero & 109,6 & 12,7 & 0,50 \\
Marzo & 85,8 & 9,6 & 0,46 \\
Abril & 44,0 & 6,8 & 0,48 \\
Mayo & 19,2 & 5,4 & 0,51 \\
\hline
\end{tabular}

(*) Cuando no se contó con información de un bote, se utilizó la media mensual para incluirlo s/i Sin información Fuente: Formularios de desembarque artesanal (SERNAPESCA) e información propia

Tabla 4. Resumen de botes en operación, salidas de pesca y captura por unidad de esfuerzo (CPUE) mensual (Isla Alejandro Selkirk).

Table 4. Summary of operating boats, fishing trips and catch per unit effort (CPUE) per month. (Alejandro Selkirk island).

\begin{tabular}{|lcccc|}
\hline Mes & Botes en operación & Salidas de pesca & & $\begin{array}{c}\text { C P U E } \\
\text { Langostas/salida }\end{array}$ \\
\hline Septiembre (1996) & 7 & & & 30,7 \\
Octubre & 6 & 75 & 329,0 & 31,8 \\
Noviembre & 8 & 43 & 227,7 & 34,2 \\
Diciembre & 8 & 92 & 393,3 & 41,3 \\
Enero (1997) & 8 & 78 & 402,6 & 36,7 \\
Febrero & 7 & 92 & 421,5 & 27,6 \\
Marzo & 7 & 55 & 216,6 & 26,6 \\
Abril & 7 & 60 & 228,0 & 2,5 \\
Mayo (*) & - & 60 & 201,3 & - \\
\hline
\end{tabular}

(*) No se realizan actividades pesqueras, al levantarse la base temporal establecida por los pescadores Fuente: Formularios de desembarque artesanal (SERNAPESCA)

Tabla 5. Resumen de botes en operación, salidas de pesca y trampas caladas por mes (Islas San Félix y San Ambrosio).

Table 5. Summary of operating boats, fishing trips and settled traps per month (San Félix and San Ambrosio islands).

\begin{tabular}{|lccc|}
\hline Mes & Botes en operación & Salidas de pesca & Trampas caladas \\
\hline Noviembre (1996) & 3 & 27 & 810 \\
Diciembre & 3 & 27 & 810 \\
\hline
\end{tabular}

Fuente: Formularios de desembarque artesanal (SERNAPESCA) 
enero, febrero $7,7 \%$, marzo $6,8 \%$, abril $6,8 \%$ y mayo $8,4 \%$. Con relación a las zonas de pesca, se determinó la menor proporción de ejemplares comerciales en la zona 3 y la mayor en la zona 10, con una media general del 7,5\%. Con relación a la profundidad, se estableció como tendencia general una mayor proporción de ejemplares comerciales en los rangos de mayor profundidad (Tablas 9 y 10).

\section{Distribución del esfuerzo}

Como una manera de visualizar la distribución de las trampas en torno a las islas Robinson Crusoe y Santa Clara, se georreferenciaron 800 aparejos sobre la carta de esas islas (Fig. 2). Estos puntos correspondieron a caladeros utilizados por los pescadores para la extracción de la langosta, lo que permite comprobar la concentración de trampas que se produce en ciertas áreas y zonas de pesca. $\mathrm{Al}$ analizar globalmente la información recopilada en torno a estas islas, se determinó que el mayor número de trampas fue calado en la zona $6(30,9 \%)$, al sur-oeste de la isla Santa Clara, en contraposición al menor número de aparejos utilizados en las zonas 1 (Bahía
Cumberland) y 2 (Puerto Francés), al noreste de la isla Robinson Crusoe (Tabla 7).

Respecto a la distribución de las trampas por rangos de profundidad, se determinó que en enerofebrero los aparejos se distribuyeron principalmente entre 29 y $165 \mathrm{~m}$, con la mayor cantidad $(50,6 \%)$ entre 84 y 137 m. En marzo-abril las trampas se encontraron distribuidas principalmente entre 84 y $165 \mathrm{~m}$, con la mayor proporción en el estrato 112$137 \mathrm{~m}$. En mayo, los aparejos se concentraron entre 112 y 165 m (91,7\%), con la mayor cantidad en el rango $112-137 \mathrm{~m}(51,3 \%)$. Estos resultados evidencian como los aparejos van siendo calados a mayor profundidad en el transcurso de la temporada de pesca (Tabla 8).

\section{Análisis de los rendimientos}

Entre enero y abril de 1997 se observó un incremento gradual en los rendimientos promedio mensuales de ejemplares totales (comerciales + no comerciales), con los valores más altos en marzo y abril (7,72-7,84 langostas/trampa) y un valor promedio general para el período analizado de 7,33 lan-

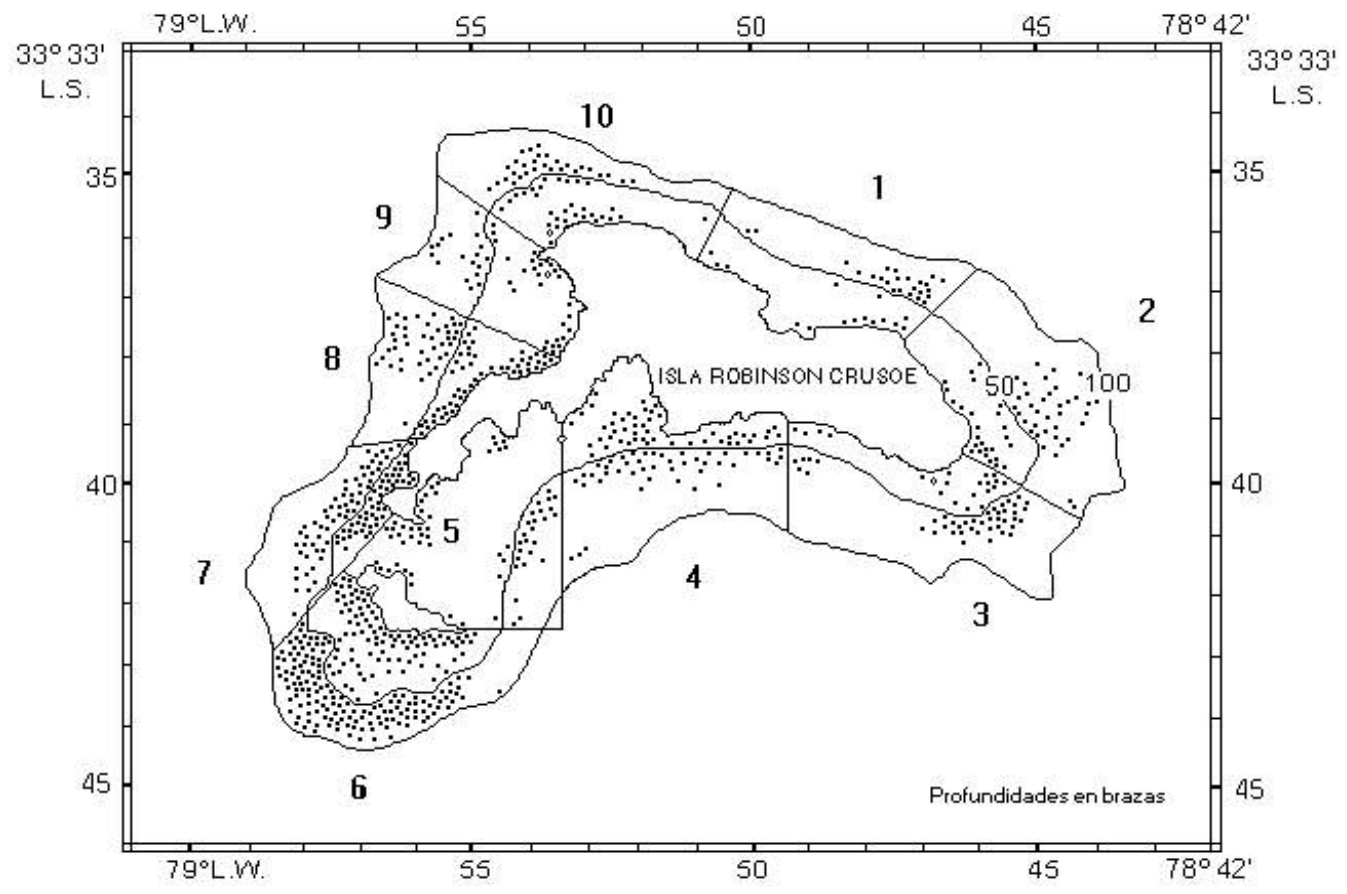

Figura 2. Ubicación de las trampas langosteras en torno a las islas Robinson Crusoe y Santa Clara.

Figure 2. Location of lobster traps around Robinson Crusoe and Santa Clara islands. 
Tabla 6. Captura por unidad de esfuerzo (CPUE) mensual por bote en operación, salida de pesca y trampa calada (Islas San Félix y San Ambrosio).

Table 6. Monthly catch per unit of effort (CPUE) by operating boat, fishing trip and settled trap (San Felix and San Ambrosio islands).

\begin{tabular}{|lccc|}
\hline Mes & Langostas/bote & CPUE \\
& & Langostas/salida & Langostas/trampa calada \\
\hline Noviembre (1996) & 1.989 & 221 & 7,37 \\
Diciembre & 1.143 & 127 & 4,23 \\
\hline
\end{tabular}

Fuente: Formularios de desembarque artesanal (SERNAPESCA)

Tabla 7. Número de trampas caladas por zona de pesca consideradas para el análisis del rendimiento (Islas Robinson Crusoe y Santa Clara).

Table 7. Number of settled traps by fishing zone considered for the yield analysis (Robinson Crusoe and Santa Clara islands).

\begin{tabular}{|lccccccccccccc|}
\hline Mes & \multicolumn{10}{c}{ Zona de pesca } & \multicolumn{1}{c|}{ Total } \\
\hline & & 1 & 2 & 3 & 4 & 5 & 6 & 7 & 8 & 9 & 10 & \\
\hline \multirow{2}{*}{ Enero } & $\mathrm{n}$ & 31 & 57 & 51 & 67 & 66 & 169 & 66 & 39 & 23 & 26 & 595 \\
& $\%$ & 5,2 & 9,6 & 8,6 & 11,3 & 11,1 & 28,4 & 11,1 & 6,6 & 3,9 & 4,4 & \\
& & & & & & & & & & & & \\
Febrero & $\mathrm{n}$ & 11 & 11 & 40 & 74 & 27 & 59 & 40 & 33 & 22 & 27 & 344 \\
& $\%$ & 3,2 & 3,2 & 11,6 & 21,5 & 7,8 & 17,2 & 11,6 & 9,6 & 6,4 & 7,8 & \\
& & & & & & & & & & & & \\
Marzo & $\mathrm{n}$ & 39 & 13 & 44 & 94 & 23 & 161 & 74 & 29 & 27 & 28 & 532 \\
& $\%$ & 7,3 & 2,4 & 8,3 & 17,7 & 4,3 & 30,3 & 13,9 & 5,5 & 5,1 & 5,3 & \\
\multirow{4}{*}{ Abril } & $\mathrm{n}$ & 74 & 44 & 106 & 115 & 46 & 336 & 178 & 86 & 78 & 49 & 1.112 \\
& $\%$ & 6,7 & 4,0 & 9,5 & 10,3 & 4,1 & 30,2 & 16,0 & 7,7 & 7,0 & 4,4 & \\
& & & & & & & & & & & & \\
Mayo & $\mathrm{n}$ & 18 & 28 & 30 & 35 & 20 & 241 & 77 & 29 & 33 & 32 & 543 \\
& $\%$ & 3,3 & 5,2 & 5,5 & 6,4 & 3,7 & 44,4 & 14,2 & 5,3 & 6,1 & 5,9 & \\
\hline \multirow{2}{*}{ Total } & $\mathrm{n}$ & 173 & 153 & 271 & 385 & 182 & 966 & 435 & 216 & 183 & 162 & 3.126 \\
& $\%$ & 5,5 & 4,9 & 8,7 & 12,3 & 5,8 & 30,9 & 13,9 & 6,9 & 5,9 & 5,2 & \\
\hline
\end{tabular}

gostas/trampa. Con relación a las zonas, los mayores rendimientos se registraron en las zonas 3 (Playa Larga) y 4 (Bahía Villagra) (Tabla 11).

Por otro lado, la CPUE promedio global de ejemplares comerciales fue de 0,55 langostas/trampa, alcanzándose el valor máximo en mayo $(0,59$ langostas/trampa) y mínimos en febrero, marzo y abril (0,53 langostas/trampa). Los ejemplares no comerciales (ejemplares bajo la talla mínima y hembras ovíferas), presentaron un rendimiento promedio global de 6,78 langostas/trampa, con un rango entre 6,13 y 7,31 langostas/trampa, obtenidos en enero y abril, respectivamente (Tabla 11).

El rendimiento por zona de pesca, presentó los valores promedios más altos de ejemplares comerciales $(>0,55$ langostas/trampa) en las zonas $4(\mathrm{Ba}-$ hía Villagra), 5 (La Canal), 6 (Santa Clara) y 10 (La Vaquería), y la cifra más baja en la zona 3 (Playa 
Larga). Al comparar los rendimientos de la fracción no comercial por zona de pesca, se determinó que las zonas 3 (Playa Larga) y 4 (Bahía Villagra) presentaron los mayores registros, con rendimientos de 8,00 y 8,26 langostas/trampa. Por el contrario, en la zona 10 (La Vaquería) se obtuvo el menor rendimiento, con sólo 5,31 langostas/trampa.

Por otra parte, se observó que el rendimiento de ejemplares comerciales tiende a aumentar hacia los estratos más profundos: 0,30 langostas/trampa en 29-55 m, llegando al máximo de 0,58 langostas/ trampa en el rango 112-137 m. En el caso de los ejemplares no comerciales, el mayor rendimiento (7,08 langostas/trampa) se obtuvo en el rango 112$137 \mathrm{~m}$. Los rendimientos obtenidos en los estratos bajo los $167 \mathrm{~m}$ son difíciles de interpretar, debido al reducido número de muestras disponibles en esas categorías (Tabla 12).

El análisis estadístico realizado a los rendimientos de ejemplares comerciales por zona de pesca (zonas 1 a 10), no muestra diferencias significativas $(\mathrm{P}>0,05)$ tanto entre los meses considerados como entre las diferentes zonas de pesca. Por otra parte, la información disponible sólo permitió analizar estadísticamente las CPUE por profundidad en 5 de los 9 estratos considerados ( 29 a $165 \mathrm{~m}$ ), determinándose en este caso diferencias significativas $(\mathrm{P}<0,05)$, que confirman que en los estratos más profundos se obtienen las CPUE más altas.

\section{DISCUSIÓN}

Al realizar la presente investigación, se ha logrado reunir por cuarta vez información de esfuerzo, captura y captura por unidad de esfuerzo de las actividades extractivas realizadas por los pescadores artesanales que operan en torno a las islas Robinson Crusoe y Santa Clara. Así, también, la recolección de registros en forma simultánea de las operaciones realizadas en la isla Alejandro Selkirk e islas Desventuradas permitió disponer en esta oportunidad de antecedentes suficientes para comparar los rendimientos entre estas islas.

Con respecto a la distribución del esfuerzo, se obtuvo un resultado semejante al estimado en años anteriores, debido a la forma tradicional con que se trabaja en esta pesquería. Así, el número de 40 chalupas de alta mar en operación durante 1997 en las islas Robinson Crusoe y Santa Clara, es compara-

Tabla 8. Número de trampas caladas por estrato de profundidad consideradas para el análisis del rendimiento (Islas Robinson Crusoe y Santa Clara).

Table 8. Number of settled traps by depth stratum considered for the yield analysis (Robinson Crusoe and Santa Clara islands).

\begin{tabular}{|c|c|c|c|c|c|c|c|c|c|c|c|}
\hline \multirow{2}{*}{\multicolumn{2}{|c|}{ Mes }} & \multicolumn{9}{|c|}{ Estrato de profundidad } & \multirow{3}{*}{$\begin{array}{r}\text { Total } \\
595\end{array}$} \\
\hline & & \multirow{3}{*}{$\begin{array}{c}0-27 \mathrm{~m} \\
0-15 \mathrm{bz}\end{array} \mid \begin{array}{c}6 \\
1,0\end{array}$} & \multirow{3}{*}{$\begin{array}{c}29-55 \mathrm{~m} \\
16-30 \mathrm{bz}\end{array}$} & \multirow{3}{*}{$\begin{array}{c}\begin{array}{c}57-82 \mathrm{~m} \\
31-45 \mathrm{bz}\end{array} \\
163 \\
27,4\end{array}$} & \multirow{3}{*}{$\begin{array}{c}84-110 \mathrm{~m} \\
46-60 \mathrm{bz}\end{array}$} & \multirow{2}{*}{$\begin{array}{c}112-137 \mathrm{~m} \\
61-75 \mathrm{bz} \\
120\end{array}$} & \multirow{3}{*}{$\begin{array}{c}\begin{array}{c}139-165 \mathrm{~m} \\
76-90 \mathrm{bz}\end{array} \\
55 \\
9,2\end{array}$} & $\begin{array}{l}167-192 \mathrm{~m} \\
91-105 \mathrm{bz}\end{array}$ & $\begin{array}{l}194-220 \mathrm{~m} \\
106-120 \mathrm{bz}\end{array}$ & $\begin{array}{l}222-247 \mathrm{~m} \\
121-135 \mathrm{bz}\end{array}$ & \\
\hline Enero & $\mathrm{n}$ & & & & & & & & & & \\
\hline & $\%$ & & & & & 20,2 & & & & & \\
\hline Febrero & $\mathrm{n}$ & & 14 & 83 & 91 & 122 & 34 & & & & 344 \\
\hline & $\%$ & & 4,1 & 24,1 & 26,5 & 35,5 & 9,9 & & & & \\
\hline Marzo & $\mathrm{n}$ & & 4 & 40 & 126 & 239 & 115 & 6 & 1 & 1 & 532 \\
\hline & $\%$ & & 0,8 & 7,5 & 23,7 & 44,9 & 21,6 & 1,1 & 0,2 & 0,2 & \\
\hline Abril & $\mathrm{n}$ & & 12 & 44 & 156 & 475 & 418 & 7 & & & 1.112 \\
\hline & $\%$ & & 1,1 & 4,0 & 14,0 & 42,7 & 37,6 & 0,6 & & & \\
\hline Mayo & $\mathrm{n}$ & & 2 & 3 & 40 & 291 & 207 & & & & 543 \\
\hline & $\%$ & & 0,4 & 0,6 & 7,4 & 53,6 & 38,1 & & & & \\
\hline Total & $\mathrm{n}$ & 6 & 102 & 333 & 594 & 1.247 & 829 & 13 & 1 & 1 & 3.126 \\
\hline & $\%$ & 0,2 & 3,3 & 10,7 & 19,0 & 39,9 & 26,5 & 0,4 & 0,0 & 0,0 & \\
\hline
\end{tabular}


Tabla 9. Porcentaje de ejemplares comerciales en las capturas por zona de pesca durante el período enero-mayo 1997.

Table 9. Percentage of commercial specimens in the catch by fishing zone during the January-May 1997 period.

\begin{tabular}{|cccccccccc|c|}
\hline \multicolumn{10}{c|}{ Zona de pesca } & Total \\
\hline 1 & 2 & 3 & 4 & 5 & 6 & 7 & 8 & 9 & 10 & \\
\hline 7,3 & 7,7 & 4,8 & 7,5 & 7,3 & 8,0 & 7,7 & 8,1 & 6,5 & 9,7 & 7,5 \\
\hline
\end{tabular}

Tabla 10. Porcentaje de ejemplares comerciales en las capturas por estrato de profundidad durante el período enero-mayo 1997.

Table 10. Percentage of commercial specimens in the catch by depth stratum during the January-May 1997 period.

\begin{tabular}{|c|c|c|c|c|c|c|c|c|c|}
\hline \multicolumn{9}{|c|}{ Estrato de profundidad } & \multirow[t]{2}{*}{ Total } \\
\hline $\begin{array}{l}0-27 \mathrm{~m} \\
0-15 \mathrm{bz}\end{array}$ & $\begin{array}{l}29-55 \mathrm{~m} \\
16-30 \mathrm{bz}\end{array}$ & $\begin{array}{l}57-82 \mathrm{~m} \\
31-45 \mathrm{bz}\end{array}$ & $\begin{array}{l}84-110 \mathrm{~m} \\
46-60 \mathrm{bz}\end{array}$ & $\begin{array}{c}112-137 \mathrm{~m} \\
61-75 \mathrm{bz}\end{array}$ & $\begin{array}{c}139-165 \mathrm{~m} \\
76-90 \mathrm{bz}\end{array}$ & $\begin{array}{l}167-192 \mathrm{~m} \\
91-105 \mathrm{bz}\end{array}$ & $\begin{array}{l}194-220 \mathrm{~m} \\
106-120 \mathrm{bz}\end{array}$ & $\begin{array}{l}222-247 \mathrm{~m} \\
121-135 \mathrm{bz}\end{array}$ & \\
\hline 0,0 & 4,6 & 6,9 & 7,8 & 7,6 & 7,6 & 8,0 & 14,3 & 0,0 & 7,5 \\
\hline
\end{tabular}

Tabla 11. Rendimientos promedio de ejemplares comerciales, no comerciales y totales (langostas/trampa), por zona de pesca durante el período enero-mayo 1997.

Table 11. Yields of commercial, non commercial and total (lobsters/trap), by fishing zone during the JanuaryMay 1997 period.

\begin{tabular}{|c|c|c|c|c|c|c|c|c|c|c|c|c|}
\hline \multirow[t]{2}{*}{ Mes } & \multirow[t]{2}{*}{ Fracción } & \multicolumn{10}{|c|}{ Zona de pesca } & \multirow[t]{2}{*}{ Total } \\
\hline & & 1 & 2 & 3 & 4 & 5 & 6 & 7 & 8 & 9 & 10 & \\
\hline \multirow[t]{3}{*}{ Enero } & Comercial & 0,35 & 0,61 & 0,43 & 0,82 & 0,61 & 0,58 & 0,56 & 0,62 & 0,26 & 0,23 & 0,56 \\
\hline & No comercial & 4,26 & 6,19 & 7,18 & 7,40 & 7,48 & 6,43 & 4,88 & 5,51 & 4,35 & 3,23 & 6,13 \\
\hline & Total & 4,61 & 6,81 & 7,61 & 8,22 & 8,09 & 7,01 & 5,44 & 6,13 & 4,61 & 3,46 & 6,69 \\
\hline \multirow[t]{3}{*}{ Febrero } & Comercial & 0,36 & 0,91 & 0,38 & 0,53 & 0,30 & 0,51 & 0,65 & 0,58 & 0,23 & 1,00 & 0,53 \\
\hline & No comercial & 4,18 & 6,00 & 10,13 & 7,30 & 5,56 & 5,31 & 9,20 & 3,42 & 3,77 & 4,26 & 6,39 \\
\hline & Total & 4,55 & 6,91 & 10,50 & 7,82 & 5,85 & 5,81 & 9,85 & 4,00 & 4,00 & 5,26 & 6,92 \\
\hline \multirow[t]{3}{*}{ Marzo } & Comercial & 0,64 & 0,38 & 0,39 & 0,61 & 0,57 & 0,53 & 0,49 & 0,31 & 0,56 & 0,68 & 0,53 \\
\hline & No comercial & 5,49 & 6,31 & 9,93 & 11,02 & 7,91 & 5,81 & 5,14 & 4,59 & 9,04 & 6,54 & 7,19 \\
\hline & Total & 6,13 & 6,69 & 10,32 & 11,63 & 8,48 & 6,34 & 5,62 & 4,90 & 9,59 & 7,21 & 7,72 \\
\hline \multirow[t]{3}{*}{ Abril } & Comercial & 0,42 & 0,41 & 0,40 & 0,71 & 0,61 & 0,61 & 0,49 & 0,48 & 0,46 & 0,39 & 0,53 \\
\hline & No comercial & 7,16 & 7,41 & 7,17 & 7,70 & 8,96 & 7,65 & 7,13 & 5,94 & 7,24 & 6,08 & 7,31 \\
\hline & Total & 7,58 & 7,82 & 7,57 & 8,41 & 9,57 & 8,26 & 7,62 & 6,42 & 7,71 & 6,47 & 7,84 \\
\hline \multirow[t]{3}{*}{ Mayo } & Comercial & 0,67 & 0,50 & 0,47 & 0,71 & 0,80 & 0,58 & 0,65 & 0,31 & 0,67 & 0,66 & 0,59 \\
\hline & No comercial & 7,28 & 5,68 & 6,67 & 6,43 & 4,95 & 6,24 & 6,57 & 6,17 & 6,79 & 5,66 & 6,28 \\
\hline & Total & 7,94 & 6,18 & 7,13 & 7,14 & 5,75 & 6,83 & 7,22 & 6,48 & 7,45 & 6,31 & 6,87 \\
\hline \multirow[t]{3}{*}{ Total } & Comercial & 0,48 & 0,54 & 0,41 & 0,67 & 0,58 & 0,58 & 0,54 & 0,47 & 0,46 & 0,57 & 0,55 \\
\hline & No comercial & 6,09 & 6,44 & 8,00 & 8,26 & 7,35 & 6,64 & 6,54 & 5,33 & 6,64 & 5,31 & 6,78 \\
\hline & Total & 6,57 & 6,98 & 8,41 & 8,94 & 7,92 & 7,21 & 7,08 & 5,80 & 7,10 & 5,88 & 7,33 \\
\hline
\end{tabular}


Tabla 12. Rendimientos de ejemplares comerciales, no comerciales y totales (langostas/trampa), por estrato de profundidad durante el período enero-mayo 1997. Table 12. Yields of commercial, non commercial and total (lobsters/trap) by depth stratrum during the January-May 1997 period.

\begin{tabular}{|c|c|c|c|c|c|c|c|c|c|c|c|}
\hline \multirow[b]{2}{*}{ Mes } & \multirow[b]{2}{*}{ Fracción } & \multicolumn{10}{|c|}{ Estrato de profundidad } \\
\hline & & $\begin{array}{l}0-27 \mathrm{~m} \\
0-15 \mathrm{bz}\end{array}$ & $\begin{array}{l}29-55 \mathrm{~m} \\
16-30 \mathrm{bz}\end{array}$ & $\begin{array}{l}57-82 \mathrm{~m} \\
31-45 \mathrm{bz}\end{array}$ & $\begin{array}{l}84-110 \mathrm{~m} \\
46-60 \mathrm{bz}\end{array}$ & $\begin{array}{c}112-137 \mathrm{~m} \\
61-75 \mathrm{bz}\end{array}$ & $\begin{array}{c}139-165 \mathrm{~m} \\
76-90 \mathrm{bz}\end{array}$ & $\begin{array}{l}167-192 \mathrm{~m} \\
91-105 \mathrm{bz}\end{array}$ & $\begin{array}{l}194-220 \mathrm{~m} \\
106-120 \mathrm{bz}\end{array}$ & $\begin{array}{ll}222-247 & \mathrm{~m} \\
121-135 & \mathrm{bz}\end{array}$ & Total \\
\hline \multirow[t]{3}{*}{ Enero } & Comercial & - & 0,39 & 0,48 & 0,66 & 0,63 & 0,62 & - & - & - & 0,56 \\
\hline & No comercial & 7,67 & 6,24 & 6,77 & 5,6 & 6,67 & 4,51 & - & - & - & 6,13 \\
\hline & Total & 7,67 & 6,63 & 7,25 & 6,26 & 7,3 & 5,13 & - & - & - & 6,69 \\
\hline \multirow[t]{3}{*}{ Febrero } & Comercial & - & 0,14 & 0,46 & 0,35 & 0,75 & 0,59 & - & - & - & 0,53 \\
\hline & No comercial & - & 6,36 & 5,71 & 6,31 & 7,17 & 5,5 & - & - & - & 6,39 \\
\hline & Total & - & 6,5 & 6,17 & 6,66 & 7,92 & 6,09 & - & - & - & 6,92 \\
\hline \multirow[t]{3}{*}{ Marzo } & Comercial & - & 0,25 & 0,33 & 0,51 & 0,52 & 0,64 & 0,67 & 1,00 & - & 0,53 \\
\hline & No comercial & - & 6,25 & 5,4 & 7,45 & 7,28 & 7,47 & 6,33 & 6,00 & 3,00 & 7,19 \\
\hline & Total & - & 6,5 & 5,73 & 7,96 & 7,8 & 8,11 & 7 & 7,00 & 3,00 & 7,72 \\
\hline \multirow[t]{3}{*}{ Abril } & Comercial & - & 0,08 & 0,45 & 0,47 & 0,57 & 0,52 & 0,57 & - & - & 0,53 \\
\hline & No comercial & - & 6,67 & 6,02 & 5,81 & 7,76 & 7,5 & 7,71 & - & - & 7,31 \\
\hline & Total & - & 6,75 & 6,47 & 6,28 & 8,33 & 8,02 & 8,28 & - & - & 7,84 \\
\hline \multirow[t]{3}{*}{ Mayo } & Comercial & - & 0,00 & 1,67 & 0,7 & 0,55 & 0,62 & - & - & - & 0,59 \\
\hline & No comercial & - & 5,00 & 6,33 & 7,45 & 5,95 & 6,53 & - & - & - & 6,28 \\
\hline & Total & - & 5,00 & 8,00 & 8,15 & 6,50 & 7,15 & - & - & - & 6,87 \\
\hline \multirow[t]{3}{*}{ Total } & Comercial & - & 0,30 & 0,46 & 0,53 & 0,58 & 0,57 & 0,62 & 1,00 & - & 0,55 \\
\hline & No comercial & 7,67 & 6,28 & 6,24 & 6,28 & 7,08 & 6,97 & 7,08 & 6,00 & 3,00 & 6,78 \\
\hline & Total & 7,67 & 6,58 & 6,70 & 6,81 & 7,66 & 7,54 & 7,70 & 7,00 & 3,00 & 7,33 \\
\hline
\end{tabular}

Bloque utilizado para el análisis estadístico de las capturas comerciales 
ble con las 36 embarcaciones que contabilizaron Arana y Melo (1973) en la temporada de pesca 19711972 y a las 41 embarcaciones registradas por Lobell et al. (1947), durante 1946-1947

Por otra parte, se estableció que el número máximo de trampas empleadas por temporada ha sido incrementado respecto a años anteriores, contabilizándose en diciembre de 1996 un total de 1.066 trampas en comparación a los 969 aparejos utilizados en noviembre de 1981 (Arana y Toro, 1985). El número máximo de aparejos empleados en la temporada analizada debe haber sido superior al registrado en diciembre, cuando se inició la recopilación de antecedentes en estas islas, debido a que por lo general la mayor cantidad de aparejos se encuentra en operación en noviembre de cada año.

Con relación al número de viajes mensuales de pesca, se observa una tendencia similar a la establecida en temporadas anteriores, aunque con valores ligeramente inferiores a los señalados por Arana y Toro (1985) durante las temporadas 1980-1981 y 1981-1982. Dicha diferencia estaría asociada a la cantidad de mareas que realizan los pescadores por más de un día, con el objeto de ahorrar combustible. Por otro lado, este menor número de salidas también estaría compensado por el mayor número de trampas que actualmente utiliza cada embarcación.

En el caso particular de las islas Robinson Crusoe y Santa Clara, donde operó aproximadamente el 75\% de la flota artesanal del archipiélago de Juan Fernández, se desembarcó el 49\% de las capturas totales registradas en la temporada 1996-1997. Dicha cantidad es significativamente menor a la reportada por Arana y Melo (1973), quienes determinaron que en estas islas se obtuvo el 65\% del desembarque total de 1971-1972, con aproximadamente el mismo número de embarcaciones en operación.

Al analizar los registros obtenidos en los muestreos realizados en el mar, se pudo comprobar que el esfuerzo se concentró principalmente en las zonas ubicadas al sur y sur-oeste de las islas Robinson Crusoe y Santa Clara (zonas 4, Bahía Villagra; 6, Santa Clara y 7, Bahía del Padre). Estos resultados coinciden con lo señalado previamente por Arana y Toro (1985), entre noviembre de 1980 y marzo de 1982, quienes además indican que en las zonas 4 (Bahía Villagra), 5 (La Canal) y 6 (Santa Clara) se concentró la mayor cantidad de trampas, destacándose la zona 6 donde en promedio se registró en esa temporada el $25 \%$ del total de aparejos calados mensualmente en torno a estas islas.
Con referencia a la distribución mensual de las trampas por estrato de profundidad, se estableció que las trampas son caladas al comienzo de la temporada preferentemente cerca de la costa y a partir de diciembre son desplazadas hacia aguas de mayor profundidad, concentrándose al final de la temporada cerca del $90 \%$ de los aparejos entre 112 y 165 m. Este patrón coincide igualmente con el descrito anteriormente por Arana y Toro (1985) para la temporada 1981-1982. Sin embargo en esa oportunidad, las trampas presentaron mayor dispersión batimétrica, concentrándose en el período abrilmayo, entre 84 y $165 \mathrm{~m}$.

La información recopilada en los muestreos en el mar, permitió establecer además el rendimiento de ejemplares comerciales y no comerciales durante el período enero-mayo 1997. Los resultados obtenidos para el primer grupo indican una CPUE promedio global de 0,55 langostas/trampa, valor inferior al determinado por Arana y Melo (1973) en la temporada 1971-1972 de 1,97 langostas/trampa y relativamente superior respecto al registrado por Arana y Toro (1985) en la temporada 1981-1982, equivalente a 0,48 langostas/trampa. Estos valores evidencian una relativa estabilización en los rendimientos en los últimos 15 años. A diferencia de lo señalado para la fracción comercial, el rendimiento promedio de ejemplares totales establecido en la temporada 1996-1997 (7,33 langostas/trampa) es inferior a los descritos por Arana y Melo (1973) y por Arana y Toro (1985) en las temporadas 19711972 y $1981-1982$, con 8,33 y 8,75 langostas/trampa, respectivamente.

Por otra parte, la CPUE de ejemplares comerciales determinada mensualmente, demostró ser similar entre los meses considerados en la temporada 1996-1997 (0,53-0,59 langostas/trampa). Estos resultados difieren de lo encontrado en anteriores investigaciones realizadas en este mismo archipiélago (Arana y Melo, 1973; Arana y Toro, 1985; Henríquez et al., 1985), en las que se menciona que a comienzos y finales de la temporada se logran rendimientos por trampa notoriamente inferiores, en comparación a los obtenidos en enero y febrero (Tabla 13).

Otro aspecto interesante de destacar es que a pesar de haberse indicado en anteriores trabajos una disminución progresiva a través de los años en la proporción de ejemplares comerciales sobre el total de langostas capturadas, esta situación no se ha evidenciado en esta oportunidad. Así, esta fracción 
constituyó el 5,3\% en el período 1981-1982 (Arana y Toro, 1985), mientras que en la presente temporada de los 22.919 ejemplares analizados en el mar, el $7,5 \%$ correspondió a langostas comerciales. Dado que actualmente en este recurso se pesca prácticamente sólo una clase anual (Arana y Olate, 1999), este resultado puede estar asociado a que durante la temporada 1996-1997 se estuvo ante una clase más abundante, o bien, que éstas fueron débiles en los años analizados previamente.

Con relación a la CPUE de ejemplares comerciales, los valores más altos se obtuvieron en las zonas 4, 5, 6 (sur y sur-oeste de las islas) y 10 (norte de Robinson Crusoe) ( $>0,55$ langosta/trampa), resultado que es similar al reportado por Arana y Melo (1973), quienes destacan que en torno a la isla Santa Clara (zonas 5 y 6) se registran los mayores rendimientos. Así, también, Arana y Toro (1985) señalan los mayores valores de CPUE en las zonas de pesca 4, 5, 6 y 7. No obstante lo anterior, al comparar los rendimientos globales de langostas comerciales registrados en las zonas 5 y 6 , se observan ahora valores menores $(0,3$ a 0,8 langostas/trampa) que los registrados por Arana y Melo (1973) (2,4 y
2,8 langostas/trampa), y similares a los obtenidos por Arana y Toro (1985) entre noviembre de 1980 y marzo de 1982 (0,17 y 1,0 langostas/trampa).

En esta oportunidad las CPUE de ejemplares comerciales determinadas por estrato de profundi$\mathrm{dad}$, tienden a aumentar a mayores profundidades, alcanzando su máximo valor (0,58 langostas/trampa) entre 112 y $137 \mathrm{~m}$. Si bien tal tendencia es similar a la registrada en 1981, temporada en que se obtuvo un rendimiento máximo de 1,8 langostas/trampa entre 112 y 165 m (Arana y Toro, 1985), es diferente a la obtenida anteriormente por Arana y Melo (1973) quienes reportaron que la mayor CPUE en el período enero-mayo de 1971 se localizó entre los 29 y $55 \mathrm{~m}$ de profundidad.

Así, también, es destacable que al comparar los rendimientos determinados por estrato de profundidad se determinaron diferencias significativas $(\mathrm{P}<0,05)$ en la fracción comercial. Este resultado es coincidente con lo señalado por Pavez y Arana (1982), Arana (1983), Arana y Venturini (1989) y Arana (1991-1992), autores que han demostrado que la langosta se desplaza en profundidad durante el transcurso de la temporada, concentrándose entre

Tabla 13. Rendimientos mensuales de ejemplares comerciales (langostas/trampa) obtenidos a través de muestreos en el mar (islas Robinson Crusoe y Santa Clara).

Table 13. Monthly yield of commercial specimens (lobsters/trap) obtained from samplings at sea (Robinson Crusoe and Santa Clara islands).

\begin{tabular}{|c|c|c|c|c|c|c|c|}
\hline \multirow{3}{*}{ Mes / año } & \multicolumn{7}{|c|}{ CPUE (langostas/trampas) } \\
\hline & \multicolumn{2}{|c|}{ Arana y Melo (1973) } & \multicolumn{2}{|c|}{ Arana y Toro (1985) } & \multicolumn{2}{|c|}{ Henríquez et al. (1985) } & \multirow{2}{*}{$\begin{array}{c}\begin{array}{c}\text { Presente } \\
\text { investigación }\end{array} \\
1997\end{array}$} \\
\hline & 1971 & 1972 & 1981 & 1982 & 1984 & 1985 & \\
\hline Octubre & 0,77 & & & 0,67 & 0,76 & & \\
\hline Noviembre & 0,90 & & & 0,51 & 0,72 & & \\
\hline Diciembre & 1,48 & & & 0,42 & 0,77 & & \\
\hline Enero & 2,39 & 3,13 & & 0,54 & & 0,95 & 0,56 \\
\hline Febrero & 2,42 & 2,71 & & 0,86 & & 0,84 & 0,53 \\
\hline Marzo & 1,71 & 1,42 & & 0,52 & & 0,77 & 0,53 \\
\hline Abril & $1,51 *$ & $1,50 *$ & 0,44 & & & 0,65 & 0,53 \\
\hline Mayo & & & 0,53 & & & 0,75 & 0,59 \\
\hline Junio & & & 0,16 & & & & \\
\hline Julio & & & 0,37 & & & & \\
\hline Agosto & & & 0,43 & & & & \\
\hline Septiembre & & & 0,42 & & & & \\
\hline Promedio & & & & & & & 0,55 \\
\hline
\end{tabular}

* Período abril-mayo 
los 55 y 128 m hacia el término del verano.

Por otra parte, la información disponible permite visualizar las diferencias en los rendimientos obtenidos en esos tres lugares. Estos, en una primera instancia, podrían ser atribuibles a la diferente intensidad de explotación ejercida en cada una de ellos. Así, en las islas San Félix y San Ambrosio (Islas Desventuradas) se calculó un promedio de 174 langostas/salida, en Alejandro Selkirk dicho valor varió entre 23,5 y 41,3 langostas/salida, mientras que en Robinson Crusoe-Santa Clara se registraron entre 5,4 y 12,9 langostas/salida. Otro aspecto interesante de destacar se refiere a los rendimientos establecidos en la isla Alejandro Selkirk, en la cual se obtuvo durante la temporada 1996-1997 un promedio de 31,6 langostas/salida, valor que casi duplica al determinado en la temporada $1984 / 1985$ por Henríquez et al. (1985) (Tabla 14).

Como apreciación general, se puede concluir que si bien los rendimientos generales en las islas Robinson Crusoe y Santa Clara se mantienen en niveles aproximadamente similares a los obtenidos en los últimos 20 años (Tabla 13), la mayor cantidad de trampas en uso y la más alta efectividad de las mismas (Arana, 1983), hace que en la actualidad se capturen todas las langostas factibles de pescar de acuerdo con las normas legales vigentes. Cabe hacer mención que las normas que regulan la extracción de la langosta en estas islas no ha sufrido modificación. De allí que una reducción en el esfuerzo redundaría en el transcurso del tiempo en mayores CPUE, vale decir, con una menor cantidad de trampas se podría capturar la misma o incluso una mayor cantidad de ejemplares.

De lo anterior se deduce que es recomendable incentivar que el esfuerzo de pesca aplicado en torno a estas dos islas disminuya en el futuro. Una acción de esta naturaleza permitiría en el largo plazo mantener el nivel de ingreso de los pescadores y tendería a incrementar la fracción de ejemplares de tamaño comercial en el mar. Esta medida igualmente contribuiría a mejorar los reclutamientos en los años siguientes y, por ende, aumentaría la biomasa del recurso, resguardando la población de langostas y asegurando el mantenimiento de la pesquería en el tiempo.

\section{AGRADECIMIENTOS}

Los autores expresan su agradecimiento al Sindicato de Pescadores del Archipiélago de Juan Fernández y a los pescadores de estas islas, por su

Tabla 14. Rendimientos mensuales de ejemplares comerciales (langostas/salida) obtenidos en la isla Alejandro Selkirk.

Table 14. Monthly yield of commercial specimens (lobsters/trap) obtained in Alejandro Selkirk island.

\begin{tabular}{|c|c|c|c|c|}
\hline \multirow{3}{*}{$\begin{array}{c}\text { Autor } \\
\text { Mes / Año }\end{array}$} & \multicolumn{3}{|c|}{ CPUE (langostas/salida) } & \\
\hline & \multicolumn{2}{|c|}{ Henríquez et al. (1985) * } & \multicolumn{2}{|c|}{ Presente investigación ** } \\
\hline & 1984 & 1985 & 1996 & 1997 \\
\hline Septiembre & 22,5 & & 30,7 & \\
\hline Octubre & 20,5 & & 31,8 & \\
\hline Noviembre & 12,9 & & 34,2 & \\
\hline Diciembre & 16,7 & & 41,3 & \\
\hline Enero & & 20,9 & & 36,7 \\
\hline Febrero & & 17,1 & & 27,6 \\
\hline Marzo & & 13,8 & & 26,6 \\
\hline Abril & & 9,1 & & 23,5 \\
\hline Mayo & & 10,7 & & - \\
\hline Promedio & 16, & & & \\
\hline
\end{tabular}

* Información de registros efectuados en el mar

** Información obtenida de Formularios de desembarque artesanal (SERNAPESCA) 
activa y desinteresada colaboración en la entrega de información y permitir el embarque de los técnicos para efectuar los muestreos en el mar. Igualmente se agradece a las empresas que comercializan esta especie, al facilitar la medición de los ejemplares en forma previa a su envío al continente.

\section{REFERENCIAS}

Arana,P. 1983. Estado en que se encuentra la pesquería de la langosta de Juan Fernández (Jasus frontalis). En: P. Arana (ed.). Análisis de Pesquerías Chilenas. Esc. Ciencias del Mar, UCV, Valparaíso, pp. 77-111.

Arana, P. 1985. Análisis y recomendaciones sobre medidas de regulación en la pesquería de la langosta de Juan Fernández (Jasus frontalis). En: P. Arana (ed.). Investigaciones Marinas en el Archipiélago de Juan Fernández. Esc. Ciencias del Mar, UCV, Valparaíso, pp. 291-300.

Arana, P. 1987. Perspectivas históricas y proyecciones de la actividad pesquera realizada en el Archipiélago de Juan Fernández, Chile. En: J.C. Castilla (ed.). Islas Oceánicas Chilenas: Conocimiento Científico y Necesidades de Investigaciones, Edic. Univ. Católica de Chile, pp. 319-353.

Arana, P. 1991-92. Desplazamientos batimétricos en la langosta de Juan Fernández (Jasus frontalis), determinados a través de metodologías de marcaje. Cienc. Téc. del Mar, CONA, 15: 49-75.

Arana, P. y C. Melo. 1973. La langosta de Juan Fernández. II. Pesca comercial de Jasus frontalis en las islas Robinson Crusoe y Santa Clara (19721973). Invest. Mar., Valparaíso, 4(5): 135-154.

Arana, P. y M.F. Pizarro. 1971. La langosta de Juan Fernández. I. Características morfométricas y distribución de tallas y sexos de Jasus frontalis de la isla Robinson Crusoe. Invest. Mar., Valparaíso, 2(5): 93-123.

Arana, P. y C. Olate. 2000. Composición de las capturas de la langosta de Juan Fernández (Jasus frontalis) y determinación de parámetros biológico-pesqueros durante la temporada 1996-1997. Invest. Mar., Valparaíso, 28: 83-115.
Arana, P. y C. Toro. 1985. Distribución del esfuerzo, rendimientos por trampa y composición de las capturas en la pesquería de la langosta de Juan Fernández (Jasus frontalis). En: P. Arana (ed.). Investigaciones Marinas en el Archipiélago de Juan Fernández. Esc. Ciencias del Mar, UCV, Valparaíso, pp. 157-185.

Arana, P. y V. Venturini. 1989. Crecimiento y migración de la langosta de Juan Fernández (Jasus frontalis) determinado a través de metodologías de marcaje. Informe final FONDECYT. Estud. Doc., Univ. Católica Valparaíso, 4/89: 1-75.

Henríquez, G., L. Rodríguez, C. Lea-Plaza, J. Sáteler y R. Salas. 1985. Diagnóstico de la pesquería de langosta en archipiélago de Juan Fernández. CORFO-IFOP, AP 86/6: 133 pp.

Larraín, F. y E. Yañez. 1985. Diseño de un sistema de recolección y procesamiento de datos de captura y esfuerzo para la pesquería de la langosta de Juan Fernández (Jasus frontalis, H. Milne Edwards, 1837). En: P. Arana (ed.). Investigaciones Marinas en el Archipiélago de Juan Fernández. Esc. Ciencias del Mar, UCV, Valparaíso, pp. 273-278.

Lobell, M.J., A.J. Byer, B.O. Knake y J.R. Westman. 1947. The fisheries of Chile. Present status and future possibilities. United States Fishery Mission to Chile, CORFO-US Department of the Interior, Washington, 1-454.

Montgomery, D.C. 1991. Diseño y análisis de experimentos. Grupo Editorial Iberoamericana S.A. de C.V., 589 pp.

Pavez, P. y P. Arana. 1982. Estimaciones de incrementos de talla, esquema de migración y determinación de tamaños poblacionales vulnerables de la langosta de Juan Fernández (Jasus frontalis). Informe final Subsecretaría de Pesca. Estud. Doc., Univ. Católica Valparaíso, 16/82: 1-90.

Servicio Nacional de Pesca (SERNAPESCA). 1998. Anuario estadístico de pesca 1997. Servicio Nacional de Pesca, Valparaíso, 307 pp.

Silva, M. y D. Cerda. 1984. Informe de la Comisión de estudio sobre el archipiélago de Juan Fernández: antecedentes y proposiciones de desarrollo. SERNAP-SUBPESCA, In litteris. 